\title{
TU/e EmonOWEN

\section{Near-field optical spectroscopy of localized and delocalized excitons in a single GaAs quantum wire}

\section{Citation for published version (APA):}

Intonti, F., Emiliani, V., Lienau, C., Elsaesser, T., Nötzel, R., \& Ploog, K. (2001). Near-field optical spectroscopy of localized and delocalized excitons in a single GaAs quantum wire. Physical Review B, 63(7), 075313-1/5.

[075313]. https://doi.org/10.1103/PhysRevB.63.075313

DOI:

10.1103/PhysRevB.63.075313

Document status and date:

Published: 01/01/2001

\section{Document Version:}

Publisher's PDF, also known as Version of Record (includes final page, issue and volume numbers)

\section{Please check the document version of this publication:}

- A submitted manuscript is the version of the article upon submission and before peer-review. There can be important differences between the submitted version and the official published version of record. People interested in the research are advised to contact the author for the final version of the publication, or visit the $\mathrm{DOI}$ to the publisher's website.

- The final author version and the galley proof are versions of the publication after peer review.

- The final published version features the final layout of the paper including the volume, issue and page numbers.

Link to publication

\section{General rights}

Copyright and moral rights for the publications made accessible in the public portal are retained by the authors and/or other copyright owners and it is a condition of accessing publications that users recognise and abide by the legal requirements associated with these rights.

- Users may download and print one copy of any publication from the public portal for the purpose of private study or research.

- You may not further distribute the material or use it for any profit-making activity or commercial gain

- You may freely distribute the URL identifying the publication in the public portal.

If the publication is distributed under the terms of Article 25fa of the Dutch Copyright Act, indicated by the "Taverne" license above, please follow below link for the End User Agreement:

www.tue.nl/taverne

Take down policy

If you believe that this document breaches copyright please contact us at:

openaccess@tue.nl

providing details and we will investigate your claim. 


\title{
Near-field optical spectroscopy of localized and delocalized excitons in a single GaAs quantum wire
}

\author{
Francesca Intonti, Valentina Emiliani,* Christoph Lienau, and Thomas Elsaesser \\ Max-Born-Institut für Nichtlineare Optik und Kurzzeitspektroskopie, D-12489 Berlin, Germany \\ Richard Nötzel and Klaus H. Ploog \\ Paul-Drude-Institut für Festkörperelektronik, D-10117 Berlin, Germany
}

(Received 6 November 2000; published 30 January 2001)

\begin{abstract}
Excitons in a GaAs quantum wire are studied in high-resolution photoluminescence experiments performed at a temperature of about $10 \mathrm{~K}$ with a spatial resolution of $150 \mathrm{~nm}$, and a spectral resolution of $100 \mu \mathrm{eV}$. We report an observation of quasi-one-dimensional excitons which are delocalized over a length of up to several microns along the quantum wire. Such excitons give rise to a $10-\mathrm{meV}$ broad luminescence band, representing a superposition of transitions between different delocalized states. In addition, we find a set of sharp luminescence peaks from excitons localized on a sub-150-nm length scale. Theoretical calculations of exciton states in a disordered quasi-one-dimensional potential reproduce the experimental results.
\end{abstract}

DOI: 10.1103/PhysRevB.63.075313

PACS number(s): 78.66.Fd, 07.60.Pb, 78.55.Cr

The interplay between localized and delocalized elec,tronic excitations underlies the fundamental optical and transport properties of low-dimensional systems such as conjugated polymers, ${ }^{1}$ electrons at surfaces, ${ }^{2}$ and semiconductor nanostructures. In quasi-two-dimensional (2D) semiconductor quantum wells (QW's) and quasi-one-dimensional quantum wires (QWR's), the optical spectra in the range of the fundamental band gap are determined by excitons showing a binding energy on the order of $10 \mathrm{meV}$ which increases upon reducing the dimensionality of the semiconductor. In the simplest picture, neglecting any structural disorder in a $\mathrm{QW}$ or QWR, such excitons are fully delocalized in space. In contrast to this expectation, extensive experiments with narrow QW's gave evidence of exciton localization in individual minima of a disorder potential originating from structural imperfections of the material. ${ }^{3-5}$ Recent progress in optical microprobing by microphotoluminescence ( $\mu$-PL) and near-field optical spectroscopy has allowed one to resolve the PL spectra from single localized excitons in QW's and QWR's, displaying a set of sharp emission lines ${ }^{6-8}$ when recorded with a submicron spatial resolution. The spectral envelopes and spatial properties of such line spectra, which reflect a quasi-zero-dimensional behavior of localized excitons, depend largely on the length scale of interface roughness in the particular system, and have been described by detailed theoretical models. ${ }^{5,9,10}$

So far, evidence of the coexistence of and the interplay between localized and delocalized excitonic states has remained scarce. Recent studies of thin QW's of substantial interface roughness by $\mu$-PL suggest the occurrence of both types of excitons ${ }^{11}$ Spatially resolved PL studies on different QWR structures ${ }^{12-14}$ did not show evidence of delocalized excitonic states, but suggested an optical behavior of QWR's arising from closely spaced localized, quasi-zerodimensional excitons. This makes these QWR's similar to a chain of quantum dots. It thus seems particularly important to clarify the role that delocalized, truly 1D excitonic states play for the optical and transport properties of such nanostructures.
In this paper we report a near-field PL study of excitons confined in a single GaAs QWR. By combining high spatial and spectral resolution, we resolve the characteristic emission features of excitons in a complex quasi-1D disorder potential. We demonstrate the coexistence of single localized excitons and of excitons that are delocalized over a length of up to several microns along the QWR. Our experimental results are well reproduced by theoretical calculations of excitonic states in a disordered QWR.

We investigate a GaAs/(AlGa)As coupled QWR-dot nanostructure grown by molecular-beam epitaxy on a patterned GaAs(311)A substrate. On the substrate, a 15-nmhigh mesa with sidewalls alternatingly misaligned by $\pm 30^{\circ}$ with respect to the [01-1] direction (zigzag pattern) is prepared by wet chemical etching. The QWR structure consists of a nominally 3-nm-thick GaAs QW layer clad between $50-\mathrm{nmAl}_{0.5} \mathrm{Ga}_{0.5} \mathrm{As}$ barriers. QWR's are formed due to the preferential migration of $\mathrm{Ga}$ adatoms from the mesa top and bottom toward the sidewalls of the mesa, ${ }^{15}$ resulting in a local increase of QW thickness up to $4.5 \mathrm{~nm}$ and a lateral confinement. ${ }^{16}$ Figure 1(a) shows a schematic top view of the structure in which the QWR follows the zigzag pattern. Every $4.5 \mu \mathrm{m}$ two QWR segments merge with an angle of $120^{\circ}$. Every other corner the mesa top surrounds the merging of the quantum wires with an angle of $240^{\circ}$. At these positions, an extra local accumulation of GaAs gives rise to the formation of dots. The confinement potential of this type of QWR was studied in earlier photoluminescence (PL) and photoluminescence excitations measurements. ${ }^{17,18}$ The lateral width of the confined region and the quasi-1D confinement energy of excitons are $70 \mathrm{~nm}$ and $55 \mathrm{meV}$, respectively.

A near-field scanning optical microscope for cryogenic temperatures was used in an illumination/collection geometry with combined spatial and spectral resolutions of 150 $\mathrm{nm}$ and $100 \mu \mathrm{eV}$, respectively. Spatially resolved images were recorded by scanning the probe tip over the sample. For high-resolution measurements, the sample was excited with either a HeNe laser $(1.96 \mathrm{eV})$ or a tunable narrow-band Ti: sapphire laser (bandwidth $<200 \mu \mathrm{eV}$ ). PL spectra were re- 
(a)

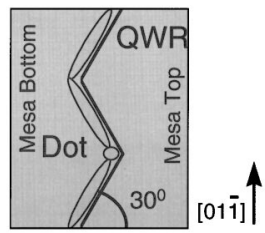

(c) $E_{\text {det }}=1.720 \mathrm{eV}$

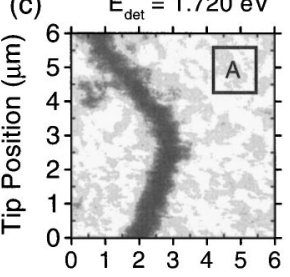

(b)

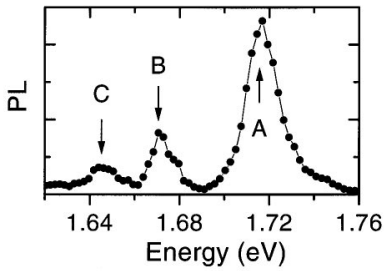

$1.668 \mathrm{eV}$

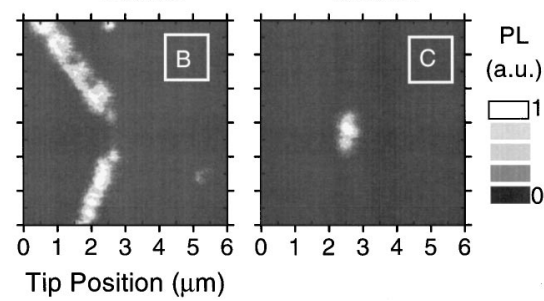

FIG. 1. (a) Schematic top view of the QWR-dot structure. The lithographic patterning of the substrate gives rise to the formation of a QWR and a dot at the corner of two intersecting sidewalls on the mesa top side. (b) Far-field 10-K PL spectrum recorded near the dot position. (c) 2D PL images recorded at the detection energies of the three peaks shown in (b).

corded at each tip position with a $f=50 \mathrm{~cm}$ monochromator in conjunction with a cooled charge-coupled device ( $T$ $=77 \mathrm{~K}$, spectral resolution $100 \mu \mathrm{eV}$ ). Aperture probes were made by chemically etching single mode optical fibers.

In Fig. 1(b), we present an overview far field PL spectrum taken in the QWR-dot area of the sample (excitation at 1.96 $\mathrm{eV}$ ). The origin of the three distinct PL peaks is evident from spatially resolved measurements where the near-field probe served for both excitation and near-field PL collection [Fig. 1(c)]. The first image, for excitation at $1.74 \mathrm{eV}$, shows the uniform distribution of the PL signal from the surrounding QW, centered at $1.720 \mathrm{eV}$. This component decreases strongly near the sidewall. The spatial distribution of the scans recorded at 1.668 and $1.650 \mathrm{eV}$ identifies QWR and dot emission, respectively (excitation $1.70 \mathrm{eV}$ ). The optical spectra of the dot structure will be discussed in detail elsewhere.
As the combined spatial and spectral resolution is decreased below $200 \mathrm{~nm} / 100 \mu \mathrm{eV}$, the broad PL bands break up into a set of sharp and intense emission peaks superimposed on a broad continuum [Fig. 2(a)]. The near-field PL spectrum was recorded at a fixed position on the QWR. The spectral widths of the PL peaks range from 200 to $350 \mu \mathrm{eV}$.

Two-dimensional near-field images, recorded at the spectral position of the sharp lines [Fig. 2(b)] show that these luminescence contributions stem from regions resolution limited in size. Thus they are attributed to the emission of localized excitons out of local potential minima that arise from monolayer-height fluctuations of the QWR profile. ${ }^{12}$ When the detection energy is tuned to the low-energy side of the broad continuum (out of the sharp resonances), the PL spatial distribution reveals the existence of regions with a larger average extension of 400-600 $\mathrm{nm}$ in diameter [Fig. $2(c)]$. For detection energies in the high-energy part of the emission band, a completely different situation appears. Here the two-dimensional images [Fig. 2(d), detection at 1.674 $\mathrm{eV}$, and excitation at $1.96 \mathrm{eV}$ indicate a uniform PL distribution delocalized along the QWR axis on a length scale of more than $2 \mu \mathrm{m}$. An even larger extension is measured in the region where the QWR's are not interrupted by dots [Fig. 2(c), excitation at $1.70 \mathrm{eV}$. In this case the spatial distribution of the PL signal extends over the whole QWR across the corner.

Delocalized PL components are observed for a broad range of detection energies. The corresponding spectral dependence is shown in Fig. 2(a) by the dotted curve. The shape of this spectrum is close to a Gaussian centered at $1.6740 \mathrm{eV}$ with a full width at half maximum of $10 \mathrm{meV}$. Similar spectra were measured in different regions of the sample, and are independent of the excitation photon energy in the range between 1.70 and $2 \mathrm{eV}$.

Figure 3 shows the power dependence of the near-field PL spectra recorded at a fixed position on the QWR. The excitation power was varied over more than three orders of magnitude with values between $P=0.002$ and $5.6 \mu \mathrm{W}$, corresponding to carrier densities between $1.4 \times 10^{2}$ and 4
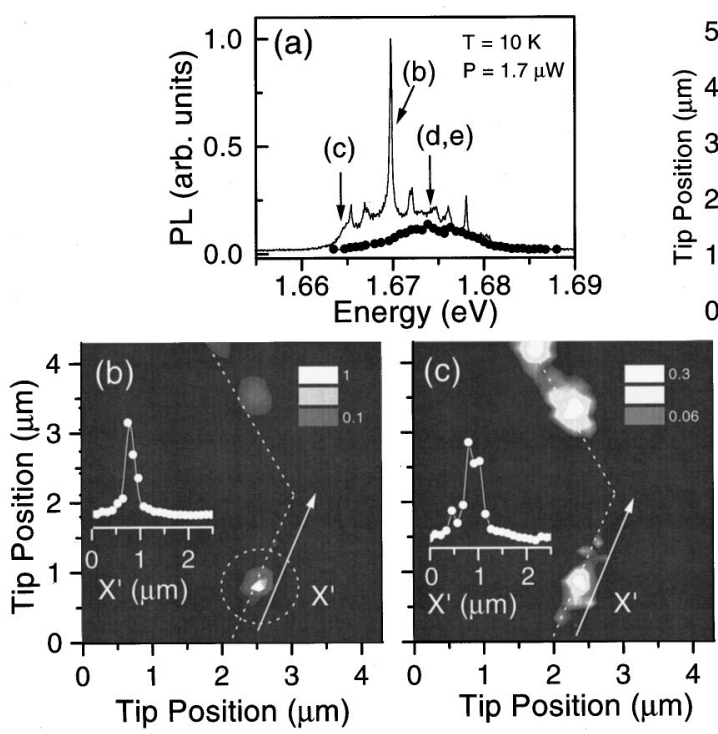
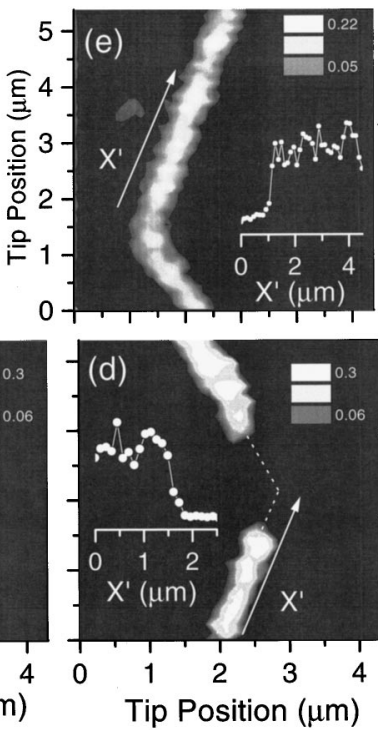

FIG. 2. (a) (Solid line) near-field PL spectrum of the QWR taken at $10 \mathrm{~K}$ at a fixed tip position corresponding to $(2.5 \mu \mathrm{m}, 0.5 \mu \mathrm{m})$ in (b) $-(\mathrm{d})$. (Circles) spectral distribution of the spatially delocalized PL contribution. (b) Two-dimensional near-field PL image taken at a fixed detection energy of $E_{\mathrm{det}}=1.6698 \mathrm{eV}$ [sharp emission peak in (a), excitation $1.96 \mathrm{eV}$ ] with a spectral resolution of $100 \mu \mathrm{eV}$. The spatial orientation of the QWRdot structure is indicated as a dotted line. Inset: cross section through the 2D image along the QWR axis $X^{\prime}$. (c) Same as (b), with $E_{\text {det }}$ $=1.6643 \mathrm{eV}$. (d) Same as (b) with $E_{\text {det }}$ $=1.674 \mathrm{eV}$ (delocalized PL component). (e) Near-field PL image taken at $E_{\mathrm{det}}=1.674 \mathrm{eV}$ (delocalized PL component) for excitation at 1.70 $\mathrm{eV}$ at a different spatial positions on the sample covering a corner where two QWRs are not interrupted by dots. 

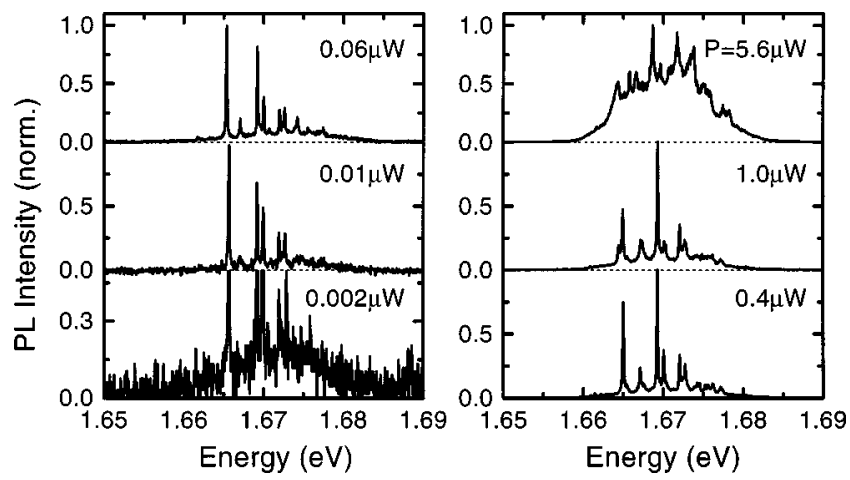

FIG. 3. Power dependence of the near-field QWR PL spectra taken at $10 \mathrm{~K}$. The excitation power is varied between $P=0.002$ and 5.6 $\mu \mathrm{W}$. Note that the broad continuum assigned to the emission of delocalized excitons contributes significantly to the PL spectra even at the lowest excitation density.

$\times 10^{5} \mathrm{~cm}^{-1}$. Note that the broad continuum contributes significantly to the PL spectra even at the lowest excitation density.

The PL spectra of the QWR observed in our experiments reveal three emission components with distinctly different spectral and spatial features: (i) a set of spectrally sharp PL lines originating from individual strongly localized exciton sites with less than 150-nm diameter, (ii) a spectrally broader component with a spatial extension of about $500 \mathrm{~nm}$, and (iii) emission from excitons which are delocalized along the QWR over a length of up to several microns and cover a $10-\mathrm{meV}$ range of emission energies. This delocalized component is present even at very low excitation densities, where only a minor fraction of the available exciton states in the QWR is populated and-thus-nonlinear effects of state and/or band filling can safely be neglected.

The occurrence of the three PL components clearly demonstrates that disorder induced local variations of the 1D confinement potential of the QWR and the interplay between localized and delocalized states play a central role for the electronic properties of this type of QWR structure. We performed a theoretical study of the excitonic spectra in order to develop a quantitative understanding of the disordered QWR structure. In this model, we consider a QWR oriented along the $x$ axis with a lateral confinement potential $V(y)$ of a width of $70 \mathrm{~nm}$ and a confinement energy of $55 \mathrm{meV}$, superimposed by a randomly generated potential [Fig. 4(a)]. The random potential is characterized by a Gaussian energy distribution of width $\sigma$ and a Gaussian correlation function in space of correlation length $L_{c}$. In the $(x, y)$ plane, excitons are subject to local energy fluctuations both along the QWR axis $(x)$ and perpendicular to it $(y)$. Following the procedure outlined in Ref. 9, the in-plane exciton center-of-mass (COM) eigenenergies and wave functions $\psi(R)[R=(X, Y)$ is the COM coordinate] are calculated by numerically solving the twodimensional Schrödinger equation for the COM motion within the local potential. As discussed in Ref. 19, this approximation of a factorized COM motion is appropriate for disorder strengths of less than $10 \mathrm{meV}$, i.e., the typical exciton binding energy $E_{X}$ in thin quantum-well and wire nano-
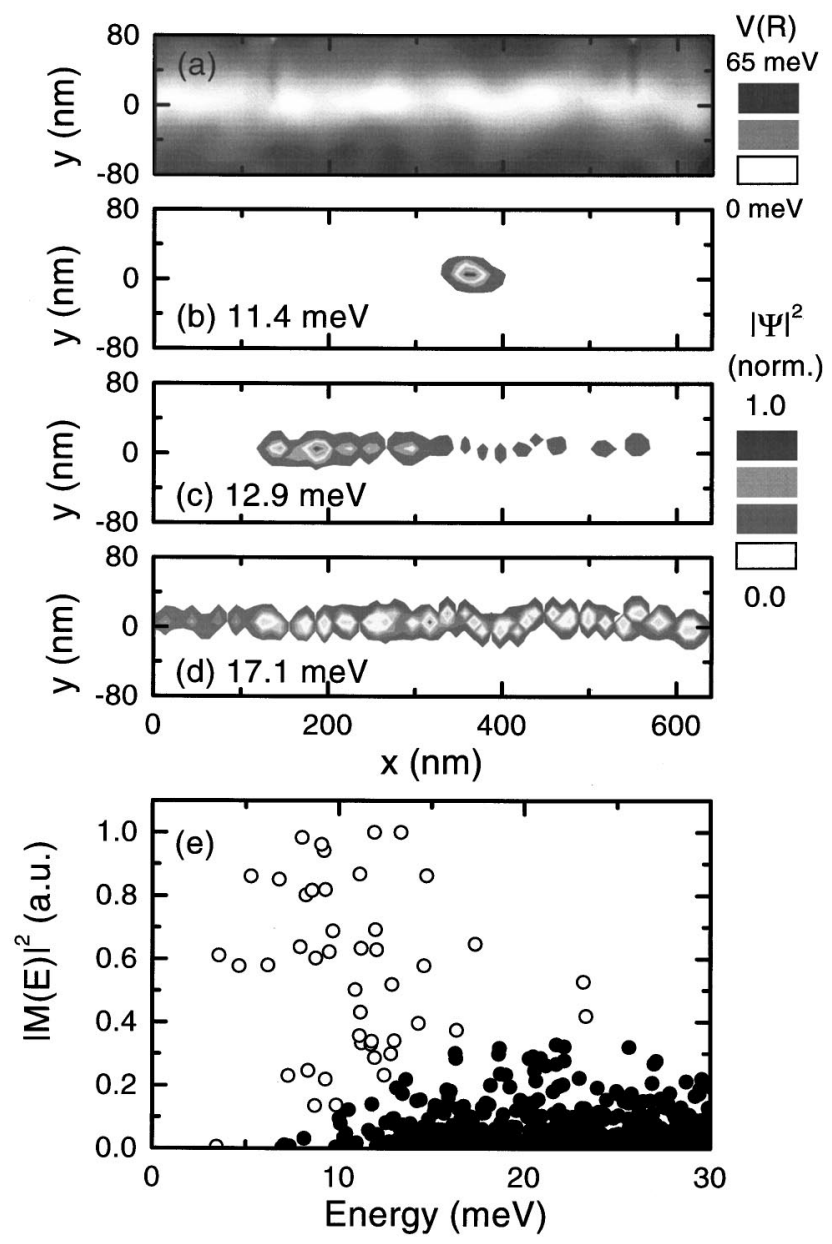

FIG. 4. (a) Potential distribution $V[R=(x, y)]$ of the exciton COM potential of a disorder QWR along $(x)$ and perpendicular $(y)$ to the QWR axis. The confinement potential is taken as $55 \mathrm{meV}$, with a lateral QWR width of $70 \mathrm{~nm}$. The disorder potential is Gauss correlated with $\sigma=5 \mathrm{meV}$ and $L_{C}=20 \mathrm{~nm}$. Probability densities $|\psi(R)|^{2}$ of representative (b) localized exciton wave function $E_{X}$ $=11.4 \mathrm{meV}$. (c) weakly delocalized excitons $E_{X}=12.9 \mathrm{meV}$, and (d) delocalized excitons $E_{X}=17.1 \mathrm{meV}$. (e) Absorption spectrum calculated for a 2- $\mu \mathrm{m}$-long QWR. The different contributions from localized (open circles) and delocalized excitons (closed circles) are highlighted.

structures. Independent experimental evidence for the validity of this assumption is obtained from the power-dependent PL spectra (Fig. 3). These spectra show no signatures of charged exciton transitions, ${ }^{20}$ that are expected in the limit $\sigma \gg E_{X}$, where electrons and holes localize separately.

For a disorder strength of a several meV, that is typical for the considered thin nanostructures, three types of $1 s$ exciton states are found in the QWR: (i) excitons localized in a single potential minimum [Fig. 4(b)] with a nearly Gaussian shaped wavefunction $\psi(R)$. The extension of $\psi(R)$ is defined by $L_{c}$ along the QWR axis $(x)$ and by the lateral width of the potential minimum along $y$. These localized excitons couple strongly to light with a matrix element $M$ $\propto D \int d R \psi(R)$ ( $D$ is the optical dipole matrix element of the excitonic $1 s$ transition) and give rise to sharp emission peaks 
in the near-field spectra. The axial density of these localized excitons is about $1 / 4 L_{c}$, and they are distributed over an energy range of roughly $2 \sigma$. Comparison with the average number and energy distribution of emission lines in Figs. 2(a) and 3 allows one to approximate the free parameters of the model as $L_{c}=20 \mathrm{~nm}$ and $\sigma=5 \mathrm{meV}$. (ii) At low transition energies, we find several eigenstates with wave functions that extend over few local potential minina, thus showing extensions between 50 and $200 \mathrm{~nm}$ [Fig. 4(c)]. Their coupling to light is reduced with respect to that of localized excitons. Comparison to experiment indicates that this class of wave functions gives rise to the emission from localized regions extending beyond the spatial resolution of our experiment in the low-energy part of the PL spectrum [Fig. 2(c)]. (iii) We find a large density of about 10 states/ $(\mu \mathrm{m} \mathrm{meV})$ that are delocalized along the QWR with wavefunction extensions between several $100 \mathrm{~nm}$ and several $\mu \mathrm{m}$. A representative wave function with an extension of about $600 \mathrm{~nm}$ is shown in Fig. 4(d). The wave functions of these eigenstates show highly complex shapes which represent disorder-induced interferences between a broad distribution of plane waves with momentum $K_{x}$ centered around an average $\left\langle K_{x}\right\rangle$. The fraction of this distribution with momentum $K_{x}<K_{\max }=E_{X} n / \hbar c\left(E_{x}\right.$ : is the exciton transition energy, $n$ : refractive index) couples to light and contributes to absorption and emission spectra with a matrix element $M$ that is considerably reduced compared to that of localized exciton states. ${ }^{21}$ In the optical spectra, these delocalized states give rise to a broad distribution of densely spaced weak resonances. If the linewidth of the individual resonances is broader than their energy separation, individual transitions are not resolved, and broad structureless bands occur in the absorption and-for sufficient thermal population of emitting states-also in the PL spectrum. We attribute the spectrally broad and spatially delocalized luminescence in our nearfield measurements [Figs. 2(d) and 2(e)] to those delocalized excitons.

In Fig. 4(e), the overall optical spectrum calculated from the model is summarized. The square of the transition moment $|M|^{2}$ is plotted as a function of energy for both localized [components (i) and (ii)] and delocalized exciton states. In agreement with our experiments, we find a broad quasicontinuous contribution from transitions between delocalized states, superimposed by individual transitions between localized levels. Both contributions occur in a similar range of transition energies. In the luminescence spectra, the relative contribution from individual eigenstates $\alpha$ is proportional to
$\left|M_{\alpha}\right|^{2} N_{\alpha}$, with $N_{\alpha}$ being the average occupation of $\alpha$. In a quasiequilibrium distribution of localized and delocalized excitons, $N_{\alpha}$ is determined by the energy of the exciton state and the temperature $T_{E}$ of the exciton gas. The overall width of the measured delocalized PL component [circles in Fig. 2(a)] is well reproduced for a value of $T_{E} \sim 30-40 \mathrm{~K}$, i.e., somewhat higher than the lattice temperature of nominally $10 \mathrm{~K}$. The elevated exciton temperature is due to the nonresonant excitation with excess energies of several $10 \mathrm{meV}$ (also see Ref. 22).

A comment should be made on the scattering processes underlying the luminescence linewidths. At low temperatures of the exciton gas, acoustic-phonon scattering represents the main inelastic relaxation mechanism. A calculation of scattering matrix elements with the exciton wave functions derived in our model gives phonon emission times of 5-10 ps from delocalized exciton states for a lattice temperature of 10 $\mathrm{K}$. This corresponds to characteristic line broadenings of 150-300 $\mu \mathrm{eV}$. Such linewidths are larger than the energy separation between delocalized states, resulting in a broad structureless emission band as observed in our measurements. Phonon absorption times of localized excitons vary strongly between the different minima of the disorder potential. They are on the order of 10 to $30 \mathrm{ps}$ at $10 \mathrm{~K}$, corresponding to linewidths of $50-150 \mu \mathrm{eV}$, similar to what is found in the PL spectra. ${ }^{23}$

In conclusion, the coexistence of localized and delocalized excitons in a quasi-1D GaAs quantum wire was observed in luminescence experiments with high spectral and spatial resolution. Excitons delocalized over a length of up to several microns give rise to a broad luminescence band. In contrast, excitons localized in minima of the quantum wire disorder potential lead to a set of narrow individual emission lines. Theoretical calculations of exciton states in a disorder potential account for this behavior, and show that the observed broad luminescence band consists of many individual transitions between delocalized states, each showing a spectral broadening larger that the mutual energy separation. Coupling between localized and delocalized states plays an important role for exciton relaxation and for excitonic transport along the quantum wire, where contributions from both ballistic and diffusive motion are expected.

This work was supported by the Deutsche Forschungsgemeinschaft (SFB296) and the European Union through the EFRE program and a Marie-Curie Fellowship (ERB4001GT975127) for one of the authors (V.E.). We gratefully acknowledge G. Cassabois for helpful discussions.
*Present address: LENS (Laboratorio Europeo di Spettroscopie non Lineari), I-50125 Firenze, Italy.

${ }^{1}$ T. A. Pham, A. Daunois, J.-C. Merle, J. Le Moigne, and J.-Y. Bigot, Phys. Rev. Lett. 74, 904 (1995).

${ }^{2}$ N.-H. Ge, C. M. Wong, R. L. Lingle Jr., J. D. McNeill, K. J. Gaffney, and C. B. Harris, Science 279, 202 (1998).

${ }^{3}$ J. Hegarty, L. Goldner, and M. D. Sturge, Phys. Rev. B 30, 7346 (1984).

${ }^{4}$ A. V. Shchegrov, D. Birkedal and J. Shah, Phys. Rev. Lett. 83, 1391 (1999), and references therein.
${ }^{5}$ V. Savona, S. Haacke, and B. Deveaud, Phys. Rev. Lett. 84, 183 (2000), and references therein.

${ }^{6}$ N. H. Bonadeo, Gang Chen, D. Gammon, D. S. Katzer, D. Park, and D. G. Steel, Phys. Rev. Lett. 81, 2759 (1998).

${ }^{7}$ H. F. Hess, E. Betzig, T. D. Harris, L. N. Pfeiffer, and K. W. West, Science 264, 1740 (1994).

${ }^{8}$ D. Gammon, E. S. Snow, B. V. Shanabrook, D. S. Katzer, and D. Park, Science 273, 87 (1996).

${ }^{9}$ E. Runge and R. Zimmermann, Adv. Solid State Phys. 38, 251 (1998). 
${ }^{10}$ D. Larousserie, Ph.D thesis, École Normale Supérieure, Paris, 1999.

${ }^{11}$ Q. Wu, R. D. Grober, D. Gammon, and D. S. Katzer, Phys. Rev. Lett. 83, 2652 (1999).

${ }^{12}$ J. Hasen, L. N. Pfeiffer, A. Pinczuk, S. He, K. W. West, and B. S. Dennis, Nature (London) 390, 54 (1997).

${ }^{13}$ J. Bellessa, V. Voliotis, R. Grousson, X. L. Wang, M. Ogura, and H. Matsuhata, Appl. Phys. Lett. 71, 2481 (1997).

${ }^{14}$ F. Vouilloz, D. Y. Oberli, F. Lelarge, B. Dwir, and E. Kapon, Solid State Commun. 108, 945 (1998).

${ }^{15}$ R. Nötzel, M. Ramsteiner, J. Menniger, A. Trampen, H.-P. Schönherr, L. Däweritz, and K. H. Ploog, Jpn. J. Appl. Phys. 35, L297 (1996).

${ }^{16}$ J. Fricke, R. Nötzel, U. Jahn, H.-P. Schönherr, L. Däweritz, and K. H. Ploog, J. Appl. Phys. 85, 3576 (1999).

${ }^{17}$ A. Richter, G. Behme, M. Süptitz, Ch. Lienau, T. Elsaesser, M. Ramsteiner, R. Nötzel, and K. H. Ploog, Phys. Rev. Lett. 79,
2145 (1997).

${ }^{18}$ Ch. Lienau, A. Richter, G. Behme, M. Süptitz, D. Heinrich, T. Elsaesser, M. Ramsteiner, R. Nötzel, and K. H. Ploog, Phys. Rev. B 58, 2045 (1998).

${ }^{19}$ R. Zimmermann, F. Grosse, and E. Runge, Pure Appl. Chem. 69, 1179 (1997).

${ }^{20}$ A. Hartmann, Y. Ducommun, E. Kapon, U. Hohenester, and E. Molinari, Phys. Rev. Lett. 84, 5648 (2000).

${ }^{21}$ D. S. Citrin, Phys. Rev. B 47, 3832 (1993).

${ }^{22}$ R. F. Schnabel, R. Zimmermann, D. Bimberg, H. Nickel, R. Lösch, and W. Schlapp, Phys. Rev. B 46, 9873 (1993).

${ }^{23}$ The calculated scattering times are sufficiently high to establish a quasiequilibrium exciton distribution close to the lattice temperature within a lumincescence decay time of about $1 \mathrm{~ns}$ (see Ref. 17). 